\title{
White Matter Alterations Associated with Pro-inflammatory Cytokines in Patients with Major Depressive Disorder
}

\author{
Jaehwa Lim', Hoyoung Sohn', Min-Soo Kwon², Borah Kim \\ ${ }^{1}$ Department of Psychiatry, ${ }^{2}$ Department of Clinical Pharmacology and Therapeutics, CHA Bundang Medical Center, CHA University School \\ of Medicine, Seongnam, Korea
}

\begin{abstract}
Objective: Regarding the neuroinflammatory theory of major depressive disorder (MDD), little is known about the effect of pro-inflammatory cytokines on white matter (WM) changes in MDD. We aimed to investigate the relationship between pro-inflammatory cytokines and WM alterations in patients with MDD.

Methods: Twenty-two patients with MDD and 22 healthy controls $(\mathrm{HC})$ were evaluated for brain imaging and pro-inflammatory cytokines including interleukin (IL)-1 $\beta$, IL-6, IL-8, interferon- $\gamma$ and tumor necrosis factor (TNF)- $\alpha$. Tract-based spatial statistics and FreeSurfer were used for brain image analysis.

Results: The levels of TNF- $\alpha$ and IL-8 were significantly higher in the MDD group than in HC. Compared to HC, lower fractional anisotropy (FA), and higher median diffusivity (MD) and radial diffusivity $(\mathrm{RD})$ values were found in the MDD group for several WM regions. Voxel-wise correlation analysis showed that the level of TNF- $\alpha$ was negatively correlated with FA, and positively correlated with MD and RD in the left body and genu of the corpus callosum, left anterior corona radiata, and left superior corona radiata.

Conclusion: Our findings suggest that TNF- $\alpha$ may play an important role in the WM alterations in depression, possibly through demyelination.
\end{abstract}

KEY WORDS: Depressive disorder, major; Cytokines; Tumor necrosis factor-alpha; White matter; Neuroimaging; Corpus callosum.

\section{INTRODUCTION}

Major depressive disorder (MDD) is a heterogeneous disease involving widespread disruptions in functional brain networks. While the most widely accepted hypothesis for the pathogenesis of MDD is the monoamine theory [1], the neurobiological mechanisms of MDD are still poorly understood. Even though various antidepressants such as selective serotonin receptor inhibitors, serotonin-norepinephrine receptor inhibitors, and norepinephrine dopamine receptor inhibitors were developed based on the monoamine theory and widely used for patients with MDD, still one-third of the patients fail to respond to

Received: June 24, 2020 / Revised: September 6, 2020

Accepted: September 16, 2020

Address for correspondence: Borah Kim

Department of Psychiatry, CHA Bundang Medical Center, $\mathrm{CHA}$

University School of Medicine, 59 Yatap-ro, Bundang-gu,

Seongnam 13496, Korea

E-mail: borahkim27@gmail.com

ORCID: https://orcid.org/0000-0001-9992-9669 antidepressant treatment [2].

Neuroinflammation has been proposed as a pathogenesis theory to explain the heterogeneity and complex feature of depression [3]. Persons who are infected by virus or bacteria experience 'sickness behavior' such as anhedonia, fatigue, anorexia, and impaired sleep similar with symptoms of depression, which are induced by pro-inflammatory cytokines - interleukin (IL)-1, IL-6 and tumor necrosis factor (TNF)- $\alpha$ [4]. Peripheral cytokines are transmitted to the brain by humoral, neural, and cellular routes $[3,5]$, which could affect neurotransmitter signaling resulting in behavioral changes. A higher prevalence of depression in patients with chronic inflammatory diseases also supports the relationship between depression and inflammation [6]. In a similar vein, several studies have reported the relationship between MDD and inflammatory cytokines [7-9], including meta-analysis studies showing that inflammatory cytokines in patients with MDD are significantly higher than healthy controls (HC) $[10,11]$. Various

(ㄷ) This is an Open-Access article distributed under the terms of the Creative Commons Attribution Non-Commercial License (http://creativecommons.org/licenses/by-nc/4.0) which permits unrestricted non-commercial use, distribution, and reproduction in any medium, provided the original work is properly cited. 
cytokines are listed, but increased levels of TNF- $\alpha$ or IL-6 in patients with MDD are the most common results [10,11]. Miller et al. [12] exhibited pro-inflammatory cytokines to act a crucial role in the pathogenesis of MDD, even suggesting peripheral blood levels of IL-1 $\beta$, IL- 6 , and TNF- $\alpha$ as biomarkers of inflammation in MDD patients.

Cytokines can disrupt the maturation of oligodendrocytes, resulting in an alteration of white matter (WM) development [13]. Oligodendrocytes, responsible for myelin- producing in the brain, are particularly vulnerable to inflammation compared to other cell types in the brain [14]. For instance, TNF- $\alpha$ can induce the death of oligodendrocytes directly by binding to TNF receptor p55 [15]. Interferon (IFN)- $\gamma$ is also known to be highly toxic to oligodendrocytes [16]. This highly susceptible nature of oligodendrocytes could explain primary demyelination due to nonspecific immune reactions, observed in many inflammatory diseases $[17,18]$.

With the development of brain imaging technology, WM microstructural changes can be detected by researching connectivity indexes such as fractional anisotropy (FA), radial diffusivity (RD), and mean diffusivity (MD), through diffusion tensor imaging (DTI) [19]. Previous DTI studies have shown that WM alterations are observed in various regions among patients with MDD [20-22]. Recently, Enhancing Neuro Imaging Genetics through MetaAnalysis consortium, a worldwide group for analyzing brain imaging, reported robust results indicating the corpus callosum (CC) and corona radiata (CR) to be the most prominent regions showing WM microstructural changes in MDD [23].

However, there is a scarce amount of research on the relationship between cytokines and WM alterations in major depression. Only one previous study showed that IL-1 $\beta$ was associated with reduced FA [24], but there was no difference in cytokine levels between patients with depression and normal controls. Therefore, this study aimed to investigate the difference in pro-inflammatory cytokine levels between patients with MDD and $\mathrm{HC}$, and whether increased levels of pro-inflammatory cytokines influenced brain WM microstructure in MDD patients.

\section{METHODS}

\section{Participants}

All patients with MDD were recruited at the $\mathrm{CHA}$
Bundang Medical Center between October 2015 and April 2017. HC subjects were also recruited for the same period by a public advertisement. Experienced psychiatrists (B.K., H.S.) performed a diagnosis of MDD based on the Mini International Neuropsychiatric Interview $[25,26]$. Patients who met the criteria of MDD according to the Diagnostic and Statistical Manual of Mental Disorders, Fourth Edition, Text Revision were enrolled. The exclusion criteria included the diagnosis of other major psychiatric disorders (e.g., schizophrenia, bipolar affective disorder, schizoaffective disorder or intellectual disability), any history of neurological disease or serious physical disease (e.g., organic mental disorder, cancer, etc.), alcohol or drug dependence, and the presence of contraindications to brain magnetic resonance imaging (MRI).

Twenty-two patients with MDD and $22 \mathrm{HC}$ participants were enrolled for this study from the participant pool of previous study [27]. All participants were $17-70$ years old, of Korean descent, and right-handed. All patients were assessed by the Hamilton Depression Rating Scale $[28,29]$ at baseline. Furthermore, all participants were scored based on the Beck Depression Inventory [30,31] and Beck Anxiety Inventory [32,33] at the time of enrollment. All subjects in this study were either medication-naïve ( $\mathrm{HC} n=22 ; \mathrm{MDD} n=14)$ or -free $(\mathrm{MDD} n=8)$ for at least 9 weeks at the time of enrollment (Supplementary Tables 1, 2; available online).

All research procedures were performed under the review of the Institutional Review Board of CHA Bundang Medical Center, CHA University (no. BD 2015-088), and all participants were provided written consent after receiving a full description of the study.

\section{Biochemical Analyses}

We collected blood samples from all participants immediately after enrollment and before the initiation of antidepressant administration in the case of patients. Venous blood samples $(6 \mathrm{ml})$ were drawn from each participant in gel tubes and processed in the laboratory immediately after collection. Serum cytokine (IL-1 $\beta$, IL-6, IL-8, IFN- $\gamma$, TNF- $\alpha$ ) levels were measured using Bio-Rad Bio-Plex ${ }^{\circledR}$ assay (Bio-Rad, Hercules, CA, USA). The cytokine levels were measured and analyzed according to the manufacturer's instruction. Any value that was below the limit of detection (LOD) for the cytokine assay was replaced with half of the LOD of the assay, which is following a ro- 
bust and well-established method [24,34].

\section{Magnetic Resonance Imaging Acquisition}

All MRI scans were acquired on a 3T GE Signa HDxt scanner (GE Healthcare, Milwaukee, WI, USA). Parameters for three-dimensional T1-weighted fast spoiled gradient recalled echo (3D T1-FSPGR) imaging were as follows: repetition time $(T R)=16 \mathrm{~ms}$, echo time $(T E)=4.3 \mathrm{~ms}$, flip angle $=10^{\circ}$, field of view $(\mathrm{FOV})=25.6 \mathrm{~cm}$, matrix $=256 \times$ 256 , slice thickness $=1.7 \mathrm{~mm}$, and isotropic voxel size $=$ $1 \times 1 \times 1 \mathrm{~mm}^{3}$. Diffusion-weighted images were acquired using an echo-planar imaging sequence with the following parameters: TR $=17,000 \mathrm{~ms}, \mathrm{TE}=108 \mathrm{~ms}, \mathrm{FOV}=24$ $\mathrm{cm}$, matrix $=144 \times 144$, slice thickness $=1.7 \mathrm{~mm}$, and voxel size $=1.67 \times 1.67 \times 1.7 \mathrm{~mm}^{3}$. We used the double-echo option to reduce the eddy-current-related distortions. To reduce the impact of spatial distortions, an 8-channel coil and an array of spatial sensitivity encoding technique (ASSET; GE Healthcare) with a sensitivity encoding speed-up factor of 2 was used. Seventy axial slices were acquired parallel to the anterior commissure-posterior commissure line covering the whole brain in 51 directions with $b=900 \mathrm{~s} / \mathrm{mm}^{2}$. We also acquired eight baseline scans with $b=0 \mathrm{~s} / \mathrm{mm}^{2}$. The least-squares method is used to estimate the DTIs from diffusion-weighted images.

\section{Image Processing}

For voxel-wise analysis of FA data, we used Tract-Based Spatial Statistics (TBSS), version 1.2, implemented in the FMRIB Software Library (FSL), version 5.0 according to standard procedures described below [35]. First, we used the FSL to perform DTI preprocessing, including skull stripping using the Brain Extraction Tool and Eddy current correction. Second, diffusion tensor values were calculated for each voxel using a least-square fit to the diffusion signal. Last, individual FA, MD, and RD metrics were derived from the three-dimensional maps using FMRIB's Diffusion Toolbox.

The FA data of all participants were aligned into the standard space (Montreal Neurologic Institute 152 standard) using the FMRIB Nonlinear Image Registration Tool. All transformed FA images were combined and applied to the original FA map, and this resulted in a standard-space version FA map. Then these FA images were averaged to create a mean FA image, which was subsequently thinned (skeletonized) to create a mean FA skeleton, taking only the centers of the WM tracts. The threshold of the skeleton was FA > 0.2 (TBSS default) to include only major fiber bundles. FA images were used to achieve non-linear registration/skeletonization stages, to estimate the projection vectors from each participant onto the mean FA skeleton, and to compare the MD and RD.

FreeSurfer software, version 6.0.0 (Massachusetts General Hospital, Boston, MA, USA, http://surfer.nmr.mgh. harvard.edu) was used to obtain the estimated total intracranial volume (eTIV) with the 3D T1-FSPGR images. The eTIV was calculated using the automated tool for measuring the volume of brain structures, which is implemented in FreeSurfer and has been validated in several studies $[36,37]$.

\section{Statistical Analysis}

Voxel by voxel statistical analysis was performed to detect regions with significant differences in FA, $M D$, and $\mathrm{RD}$ between the MDD and $\mathrm{HC}$ groups using nonparametric permutation tests with a correction for multiple comparisons with the FSL Randomise tool [38]. To achieve accurate inference, we used a permutation-based nonparametric inference within the framework of the general linear model tested with 10,000 permutations, including a full correction for multiple comparisons over space; $p<0.05$ was considered statistically significant. Multiple comparisons were corrected with threshold-free cluster enhancement [39] to avoid making an arbitrary choice of the cluster-forming threshold while preserving the sensitivity benefits of the cluster-wise correction. Furthermore, analysis of covariance was conducted to adjust the effect of other variables, including age, sex, and eTIV on the results.

Correlation analyses were conducted to investigate whether the regional differences in FA and MD, RD could be potentially associated with the cytokine levels in each diagnostic group. For this purpose, the DTI data were analyzed using the TBSS General Linear Model regression analysis, with the cytokine levels as the variable. Clusters with 100 or more contiguous voxels were only considered in the analysis to reduce the possibility of false-positive results. We used the International Consortium for Brain Mapping-DTI-81 white-matter labels atlas of Johns Hopkins University DTI-based white-matter atlases [40,41] and the Talairach atlas $[42,43]$ to identify altered regions.

An independent $t$ test was used to compare continuous 
Table 1. Characteristics of patients with MDD and HC

\begin{tabular}{|c|c|c|c|c|}
\hline Variable & $\operatorname{MDD}(n=22)$ & $\mathrm{HC}(\mathrm{n}=22)$ & $t$ & $p$ value \\
\hline Sex, male/female & $7 / 15$ & $10 / 12$ & & $0.353^{\mathrm{a}}$ \\
\hline Age $(y r)$ & $39.32 \pm 14.48$ & $39.23 \pm 11.82$ & 0.023 & 0.982 \\
\hline $\operatorname{eTIV}\left(\mathrm{mm}^{3}\right)$ & $1,455,779.23 \pm 126,962.78$ & $1,507,458.37 \pm 131,894.39$ & -1.324 & 0.193 \\
\hline IL-1 $\beta$ levels (pg/ml) & $0.48 \pm 0.06$ & $0.02 \pm 0.00$ & 2.126 & $0.046^{\mathrm{b}}$ \\
\hline IL-6 levels (pg/ml) & $0.97 \pm 3.64$ & $0.18 \pm 0.28$ & 1.012 & 0.323 \\
\hline IL-8 levels (pg/ml) & $3.27 \pm 2.01$ & $1.31 \pm 1.70$ & 3.501 & $0.001^{\mathrm{c}}$ \\
\hline IFN- $\gamma$ levels (pg/ml) & $0.27 \pm 0.47$ & $0.11 \pm 0.26$ & 1.393 & 0.173 \\
\hline TNF- $\alpha$ levels (pg/ml) & $6.83 \pm 3.12$ & $3.07 \pm 3.23$ & 3.922 & $<0.001^{\mathrm{c}}$ \\
\hline HAMD total score & $18.23 \pm 5.36$ & - & & \\
\hline BDI total score & $20.89 \pm 7.68$ & $4.81 \pm 5.04$ & 7.114 & $<0.001^{\mathrm{c}}$ \\
\hline BAI total score & $18.31 \pm 10.98$ & $2.44 \pm 3.22$ & 5.55 & $<0.001^{\mathrm{c}}$ \\
\hline
\end{tabular}

Values are presented as number only or mean \pm standard deviation.

MDD, major depressive disorder; HC, healthy controls; eTIV, estimated total intracranial volume; IL, interleukin; IFN, interferon; TNF, tumor necrosis factor; HAMD, Hamilton Depression Rating Scale; BDI, Beck Depression Inventory; BAI, Beck Anxiety Inventory.

${ }^{\mathrm{a} C}$ Chi-square test. ${ }^{\mathrm{b}} p<0.05$ without multiple comparison correction. ${ }^{\mathrm{c} B o n f e r r o n i}$ correction $p<0.01$.

variables, and the Pearson chi-squared test was used to analyze categorical variables. Multiple comparisons were performed using Bonferroni correction in the analysis of comparing cytokine levels between two groups. Statistical analysis was performed using SPSS 26.0 (IBMCo., Armonk, NY, USA), and $p<0.05$ was considered statistically significant.

\section{RESULTS}

\section{Characteristics of Study Participants}

Table 1 presents the characteristics of the study participants. There were no significant differences between patients with MDD and HC for age, sex, and eTIVs. After multiple comparison correction, TNF- $\alpha$ and IL- 8 showed significant differences between the two groups. In contrast, there were no statistically significant differences for the other cytokines such as IFN- $\gamma$ and IL- 6 between the two groups.

\section{Comparison of White Matter Connectivity between Major Depressive Disorder Patients and Healthy Controls}

The FA values obtained by TBSS analysis showed that patients with MDD have significantly lower FA than $\mathrm{HC}$ in various regions. The following regions are depicted in Figure 1: the body and genu of the CC, bilateral superior corona radiata $(\mathrm{SCR})$, bilateral anterior corona radiata (ACR), and bilateral superior longitudinal fasciculus (SLF) (Fig. 1A). Age, sex, and eTIV were included as covariates in the analysis. Significant differences between the two groups were also found for MD (Fig. 1B) and RD values (Fig. 1C).

\section{Correlation Analyses between White Matter Connectivity and Cytokine Levels}

We conducted voxel-wise correlation analyses between cytokine levels and FA, MD, and RD values of the WM clusters that showed significant group differences in each diagnostic group. Only TNF- $\alpha$ showed correlations with WM integrity but the other cytokines showed no correlation. In patients with MDD, the TNF- $\alpha$ level showed negative correlations with FA values of the following WM regions (Fig. 2A): left ACR, left SCR, body, and genu of CC. The TNF- $\alpha$ levels showed positive correlations with $\mathrm{MD}$ (Fig. 2B) and RD values (Fig. 2C) in WM regions showing negative correlations with $\mathrm{FA}$ values. In the $\mathrm{HC}$, there was no significant correlation between the level of TNF- $\alpha$ and FA, MD, or RD values.

\section{DISCUSSION}

Antidepressant treatment based on the monoamine theory, although being the current mainstream treatment of MDD, has shown to result in more than $30 \%$ of patients failing to achieve remission despite multiple treatment trials [2]. Also, the monoamine theory alone cannot fully describe the complex characteristics of depression [3]. Therefore, the identification of alternative pathogenetic mechanisms of MDD, related potential biomarkers and 


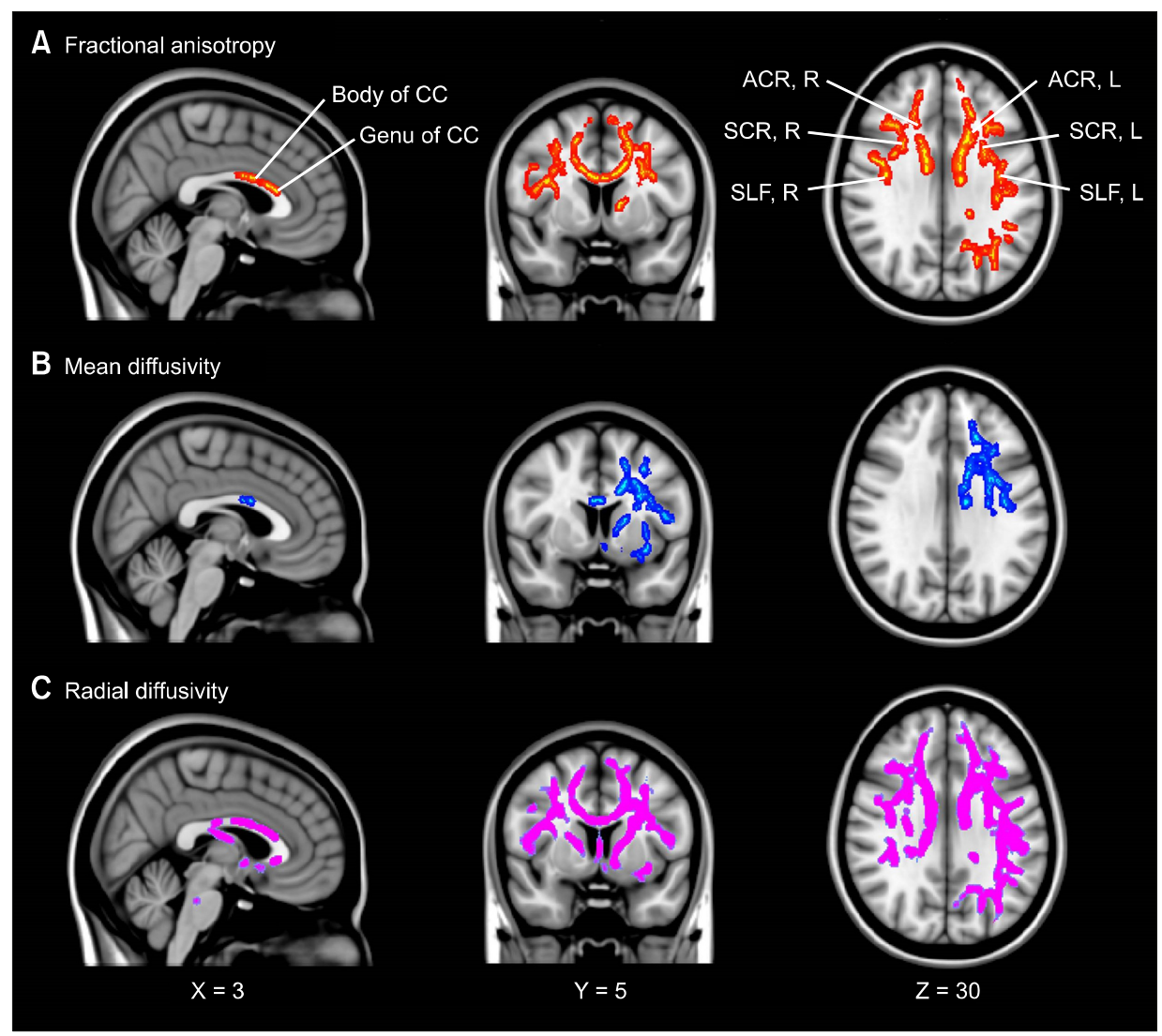

Fig. 1. Voxel-wise analysis showing significant differences (threshold-free cluster enhancement-corrected $p<0.05$ ) between patients with major depressive disorder (MDD) and healthy controls $(\mathrm{HC})$. (A) The regions showing significantly decreased fractional anisotropy in patients with MDD compared to $\mathrm{HC}$ are depicted in red-yellow. (B) The regions showing significantly increased mean diffusivity in patients with MDD compared to HC are depicted in blue-light blue. (C) The regions showing significantly increased radial diffusivity in patients with MDD compared to HC are depicted in light blue-purple. Results are shown overlaid on the Montreal Neurologic Institute 1-mm template.

$\mathrm{R}$, right; L, left; ACR, anterior corona radiata; CC, corpus callosum; SCR, superior corona radiata; SLF, superior longitudinal fasciculus.

neurobiological targets of novel therapeutic options, is needed. One of the candidate mechanisms in this regard is neuroinflammation and associated increases in cytokine levels. We hypothesized a significant difference in pro-inflammatory cytokine levels would exist between patients with MDD and $\mathrm{HC}$, and this would in turn lead to alterations in WM connectivity. We observed decreased FA values of the CC, bilateral SCR, ACR, and SLF, in patients with depression patients compared to HC. Our results are in line with a previous study published from our lab [27] and other studies reporting WM alterations in patients with MDD $[44,45]$. Our results also showed TNF- $\alpha$ levels to be negatively correlated with FA values of the left SCR, left ACR, body, and genu of CC, in the MDD patient group. To our knowledge, this is the first study to report correlations between TNF- $\alpha$ levels and WM connectivity in patients with MDD.
According to the previous DTI studies, the reduced FA values reflect WM abnormalities [20]. Also, elevations in the RD values are known to be associated with demyelination or dysmyelination, and higher MD values can be related to the increased level of tissue water often due to inflammation [46]. Thus, it can be assumed that alterations in WM regions showing the decreased FA, and increased MD and RD values are associated with tissue water increase and myelination deficits, which have been observed in patients with MDD [20] and other demyelinating diseases, such as multiple sclerosis [47].

Our results showed an increased level of pro-inflammatory cytokines, such as TNF- $\alpha$ and IL-8 in patients with MDD than $\mathrm{HC}$, which is in conjunction with other studies regarding neuroinflammation $[10,48,49]$ including a meta-analysis showing higher TNF- $\alpha$ level in MDD [11]. According to Tarkowski et al. [50], TNF- $\alpha$ is one of 


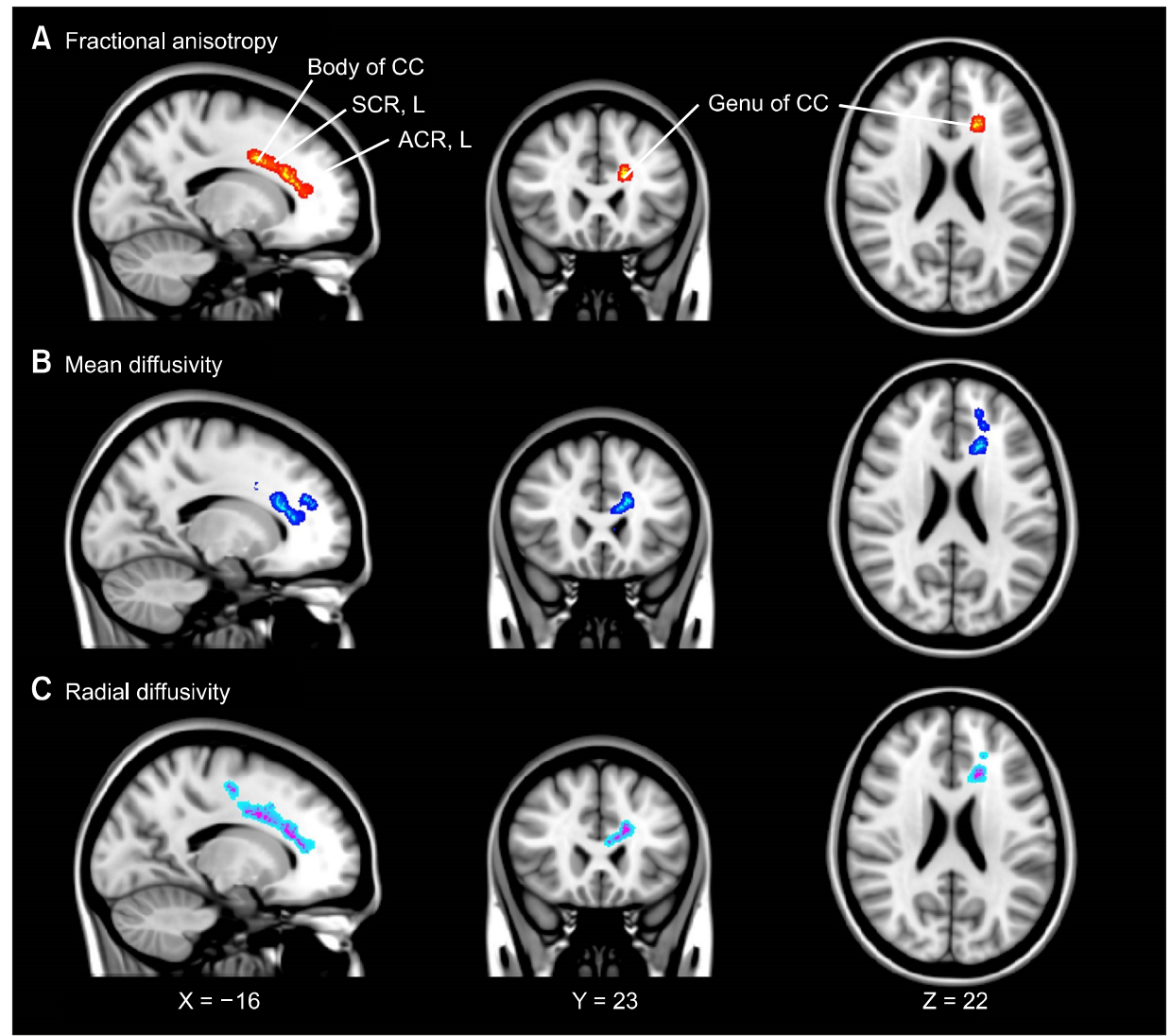

Fig. 2. Voxel-wise analysis showing significant correlations (threshold-free cluster enhancement-corrected $p<0.05$ ) between tumor necrosis factor (TNF)- $\alpha$ levels and fractional anisotropy (FA), mean diffusivity (MD), and radial diffusivity (RD) in patients with major depressive disorder (MDD). (A) The regions showing significant negative correlations between TNF- $\alpha$ levels and FA values are depicted in red-yellow. (B) The regions showing significant positive correlations between TNF- $\alpha$ levels and MD values are depicted in blue-light blue. (C) The regions showing significant positive correlations between TNF- $\alpha$ levels and RD values are depicted in light blue-purple. The voxels showing significant differences between patients with MDD and healthy controls were used for correlation analyses. Images are shown overlaid on the Montreal Neurologic Institute 1-mm template.

L, left; ACR, anterior corona radiata; CC, corpus callosum; SCR, superior corona radiate.

the key cytokines in the immune system of central nervous system (CNS). The dynamic role of TNF- $\alpha$ in the brain has been studied in other psychiatric disturbances, such as Alzheimer's disease [50] and multiple sclerosis [51], which suggested TNF- $\alpha$ might affect brain structure to progress disease through demyelination and loss of oligodendrocytes [52]. Even though the mechanism of the loss of oligodendrocytes has not completely defined yet, it has been observed that TNF- $\alpha$ induces oligodendrocyte degeneration $[15,53]$ through activation of microglia to produce Monocyte Chemoattractant Protein-1, attracting monocytes to the brain [54]. Considering the previous findings above, it can be assumed that TNF- $\alpha$ plays a crucial role in changes in oligodendrocytes, the myelinating cells of the CNS. Therefore, the current findings showing decreased FA and increased MD and RD on several WM tracts and their significant correlations with TNF- $\alpha$ level can be related to inflammation-related alterations of WM myelination in patients with MDD.

In the present findings, higher TNF- $\alpha$ is correlated with lower FA of left ACR and SCR regions in patients with MDD. $C R$ is the fan-shaped projection fibers linking the cerebral cortex to subcortical regions such as thalamus [55]. The ACR is a part of limbic-thalamo-cortical circuitry and is known to be associated with emotional regulation, one of the major affected functions in MDD [56,57]. Also, there are several pieces of research providing that microstructural change of ACR could be associated with many of core symptoms of depression by impairing communication between cortex and thalamus that is required 
for emotional regulation and executive attention [58,59]. For example, Niogi et al. [60] reported that the FA of the ACR modulates executive attention, and is also correlated with attention network task. According to Choi et al. [45], MDD patients showed reduced FA in the ACR and PCR, which suggested the disruption of emotional and cognitive control networks as the pathophysiology of MDD. Similar to our findings, lower FA, and higher MD and RD of $\mathrm{CR}$ associated with inflammation-related cytokines have been observed in patients with bipolar depression [61].

We also have shown reduced FA of the body and genu of CC is correlated with higher TNF- $\alpha$ in patients with $\mathrm{MDD}$. The $\mathrm{CC}$ is the largest commissural fibers connecting the left and right hemisphere, which develops mostly during childhood and adolescence, and keeps changing with aging [62]. The maturation of CC is mainly accomplished by axonal myelination related to the maturation of cognitive processes, and a disruption of this process may induce deficits in memory and executive functioning [63]. Especially the defect on genu of CC connecting to the prefrontal cortex was reported to contribute to the impaired inter-hemispheric connectivity, resulting in deficits in working memory and cognition [64]. Besides, the linkage among the genu of $\mathrm{CC}$, anterior cingulate, and insula is a key component of the fronto-limbic connection whose dysfunction is associated with emotional and autonomic dysregulation in depression $[57,65]$. In line with our results for WM alterations of $\mathrm{CC}$, lower FA of $\mathrm{CC}$ has been reported by other neuroimaging studies in various depression groups including late-onset depression [66], early-onset adolescent [64], and drug naïve patients [22]. Given that CC is known to be the most populated brain area by microglia [67] and oligodendrocytes, and the most vulnerable to damages [68], increased TNF- $\alpha$ could induce inflammatory changes, resulting in axonal damage by demyelination or oligodendrocyte apoptosis in the CC [69].

However, even though the IL-8 level was significantly higher in the MDD group, no significant results for the correlation analysis between IL-8 level and DTI metrics. There have been inconsistent results for the relationship between IL-8 and MDD. For example, Memon et al. [70] showed that the level of IL-8 was higher in the depression group, but it was negatively associated with the severity of depression. Also, a couple of recent meta-analyses showed no difference in the level of IL-8 between patients with MDD and HCs $[10,11]$. IL-8 is known to have both pro- or anti-inflammatory functions depending on the concentration, which implies a higher and a lower level of IL-8 might work toward opposite directions [71]. Therefore, it would be possible that IL-8 might act as both the destructive and protective roles in the possible demyelination process, which may be associated with the positive results in group comparison and the negative results for correlation analysis in this study.

There are several limitations to the study. First, the sample size is relatively small. Although the sample size in this study was within a similar range with those of other DTI imaging studies $[72,73]$, future studies with a larger sample size may be needed to confirm the findings. Second, all participants in the MDD group were medication-free, but not drug naïve. Since at the time of enrollment all participants are medication-free at least 9 weeks, we assumed that chance of medication effect to the level of cytokines or brain microstructures might be minimal. Third, this study was not designed to include other inflammatory indicators such as white blood cell counts, absolute neutrophil counts, and C-reactive protein. Even though we excluded any participants who have major physical diseases including infection or autoimmune diseases, it would be helpful to see those indicators in future studies. Lastly, our findings showed a significant correlation with cytokine levels and the WM integrity metrics, but it cannot be concluded as a causal relationship between them. We assumed pro-inflammatory cytokine levels might affect oligodendrocytes and demyelinating axons, but it may require further longitudinal studies to find out the causal relationship between pro-inflammatory cytokine and WM microstructural changes.

In conclusion, we report, for the first time, a significant association between TNF- $\alpha$ levels and WM alterations in patients with MDD. Our finding suggests that the increased level of TNF- $\alpha$ is associated with the WM integrity of CR and CC in patients with MDD. This study proposes that pro-inflammatory cytokine TNF- $\alpha$ might play an important role in the WM alterations of patients with depression. It would be needed for further studies to reveal the full function of TNF- $\alpha$ in neuroinflammation and $\mathrm{TNF}-\alpha$ as a potential biomarker in MDD. 


\section{Acknowledgments}

This research was supported by the Korean Mental Health Technology R\&D Project, Ministry of Health \& Welfare, Republic of Korea (HM15C1094). The funding sources had no further role in the study design; collection, analysis, and interpretation of data; writing of the paper; and decision to submit the paper for publication. We are thankful to Eunsoo Won, MD, Ph.D. for serving as a scientific advisor, and Min-Kyoung Kim MD, Ph.D. for technical editing of the manuscript.

\section{Conflicts of Interest}

No potential conflict of interest relevant to this article was reported.

\section{Author Contributions}

Conceptualization: Jaehwa Lim, Hoyoung Sohn, Borah Kim. Data acquisition: Jaehwa Lim, Hoyoung Sohn, Borah Kim. Formal analysis: Jaehwa Lim, Hoyoung Sohn, Min-Soo Kwon. Funding: Borah Kim. Supervision: Borah Kim. Writing original draft: Jaehwa Lim. Writing review \& editing: Jaehwa Lim, Borah Kim.

\section{ORCID}

Jaehwa Lim

Hoyoung Sohn

https://orcid.org/0000-0003-4276-3252

Min-Soo Kwon https://orcid.org/0000-0002-1856-8636

Borah Kim https://orcid.org/0000-0002-2837-881X https://orcid.org/0000-0001-9992-9669

\section{REFERENCES}

1. Hirschfeld RM. History and evolution of the monoamine hypothesis of depression. J Clin Psychiatry 2000;61 Supp/ 6:4-6.

2. Machado M, Iskedjian M, Ruiz I, Einarson TR. Remission, dropouts, and adverse drug reaction rates in major depressive disorder: a meta-analysis of head-to-head trials. Curr Med Res Opin 2006;22:1825-1837.

3. Miller $\mathrm{AH}$, Raison $\mathrm{CL}$. The role of inflammation in depression: from evolutionary imperative to modern treatment target. Nat Rev Immunol 2016;16:22-34.

4. Konsman JP, Parnet P, Dantzer R. Cytokine-induced sickness behaviour: mechanisms and implications. Trends Neurosci 2002;25:154-159.

5. Dantzer R, Konsman JP, Bluthé RM, Kelley KW. Neural and humoral pathways of communication from the immune system to the brain: parallel or convergent? Auton Neurosci 2000;85:60-65.

6. Slavich GM, Irwin MR. From stress to inflammation and major depressive disorder: a social signal transduction theory of depression. Psychol Bull 2014;140:774-815.

7. Maes M, Lambrechts J, Bosmans E, Jacobs J, Suy E, Vandervorst $\mathrm{C}$, et al. Evidence for a systemic immune activation during depression: results of leukocyte enumeration by flow cytometry in conjunction with monoclonal antibody staining. Psychol Med 1992;22:45-53.

8. Maes M, Meltzer HY, Bosmans E, Bergmans R, Vandoolaeghe $\mathrm{E}$, Ranjan $\mathrm{R}$, et al. Increased plasma concentrations of interleukin-6, soluble interleukin-6, soluble interleukin-2 and transferrin receptor in major depression. J Affect Disord 1995; 34:301-309.

9. Mikova O, Yakimova R, Bosmans E, Kenis G, Maes M. Increased serum tumor necrosis factor alpha concentrations in major depression and multiple sclerosis. Eur Neuropsychopharmacol 2001;11:203-208.

10. Dowlati Y, Herrmann N, Swardfager W, Liu H, Sham L, Reim EK, et al. A meta-analysis of cytokines in major depression. Biol Psychiatry 2010;67:446-457.

11. Köhler CA, Freitas TH, Maes M, de Andrade NQ, Liu CS, Fernandes BS, et al. Peripheral cytokine and chemokine alterations in depression: a meta-analysis of 82 studies. Acta Psychiatr Scand 2017;135:373-387.

12. Miller $\mathrm{AH}$, Maletic $\mathrm{V}$, Raison $\mathrm{CL}$. Inflammation and its discontents: the role of cytokines in the pathophysiology of major depression. Biol Psychiatry 2009;65:732-741.

13. Favrais G, van de Looij Y, Fleiss B, Ramanantsoa N, Bonnin P, Stoltenburg-Didinger G, et al. Systemic inflammation disrupts the developmental program of white matter. Ann Neurol 2011;70:550-565.

14. Deng W. Neurobiology of injury to the developing brain. Nat Rev Neurol 2010;6:328-336.

15. Jurewicz A, Matysiak M, Tybor K, Kilianek L, Raine CS, Selmaj K. Tumour necrosis factor-induced death of adult human oligodendrocytes is mediated by apoptosis inducing factor. Brain 2005;128(Pt 11):2675-2688.

16. Horiuchi $\mathrm{M}$, Itoh $\mathrm{A}$, Pleasure $\mathrm{D}$, Itoh T. MEK-ERK signaling is involved in interferon-gamma-induced death of oligodendroglial progenitor cells. J Biol Chem 2006;281:20095-20106.

17. Wisniewski HM, Bloom BR. Primary demyelination as a nonspecific consequence of a cell-mediated immune reaction. J Exp Med 1975;141:346-359.

18. Bradl M, Lassmann H. Oligodendrocytes: biology and pathology. Acta Neuropathol 2010;119:37-53.

19. Taylor WD, Hsu E, Krishnan KR, MacFall JR. Diffusion tensor imaging: background, potential, and utility in psychiatric research. Biol Psychiatry 2004;55:201-207.

20. Korgaonkar MS, Grieve SM, Koslow SH, Gabrieli JD, Gordon $\mathrm{E}$, Williams LM. Loss of white matter integrity in major depressive disorder: evidence using tract-based spatial statistical analysis of diffusion tensor imaging. Hum Brain Mapp 2011; 32:2161-2171.

21. Zuo N, Fang J, Lv X, Zhou Y, Hong Y, Li T, et al. White matter 
abnormalities in major depression: a tract-based spatial statistics and rumination study. PLoS One 2012;7:e37561.

22. Guo WB, Liu F, Xue ZM, Gao K, Wu RR, Ma CQ, et al. Altered white matter integrity in young adults with first-episode, treatment-naive, and treatment-responsive depression. Neurosci Lett 2012;522:139-144.

23. van Velzen LS, Kelly S, Isaev D, Aleman A, Aftanas LI, Bauer $\mathrm{J}$, et al. White matter disturbances in major depressive disorder: a coordinated analysis across 20 international cohorts in the ENIGMA MDD working group. Mol Psychiatry 2020; 25:1511-1525.

24. Sugimoto K, Kakeda S, Watanabe K, Katsuki A, Ueda I, Igata $\mathrm{N}$, et al. Relationship between white matter integrity and serum inflammatory cytokine levels in drug-naive patients with major depressive disorder: diffusion tensor imaging study using tract-based spatial statistics. Trans/ Psychiatry 2018;8:141.

25. Yoo SW, Kim YS, Noh JS, OH KS, Kim CH, Namkoong K, et al. Validity of Korean version of the mini-international neuropsychiatric interview. Anxiety Mood 2006;2:50-55.

26. Sheehan DV, Lecrubier $Y$, Sheehan KH, Amorim P, Janavs J, Weiller $\mathrm{E}$, et al. The Mini-International Neuropsychiatric Interview (M.I.N.I.): the development and validation of a structured diagnostic psychiatric interview for DSM-IV and ICD-10. JClin Psychiatry 1998;59 Suppl 20:22-33;quiz 34-57.

27. Sohn $\mathrm{H}$, Kwon MS, Lee SW, Oh J, Kim MK, Lee SH, et al. Effects of uric acid on the alterations of white matter connectivity in patients with major depression. Psychiatry Investig 2018;15:593-601.

28. Hamilton M. A rating scale for depression. J Neurol Neurosurg Psychiatry 1960;23:56-62.

29. Yi JS, Bae SO, Ahn YM, Park DB, Noh KS, Shin HK, et al. Validity and reliability of the Korean version of the Hamilton Depression Rating Scale(K-HDRS). I Korean Neuropsychiatr Assoc 2005;44:456-465.

30. Beck AT, Ward CH, Mendelson M, Mock J, Erbaugh J. An inventory for measuring depression. Arch Gen Psychiatry 1961; 4:561-571.

31. Lee YH, Song JY. A study of the reliability and the validity of the BDI, SDS, and MMPI-D scales. Korean J Clin Psychol 1991;10:98-113.

32. Beck AT, Epstein N, Brown G, Steer RA. An inventory for measuring clinical anxiety: psychometric properties. J Consult Clin Psychol 1988;56:893-897.

33. Yook SP, Kim ZS. A clinical study on the Korean version of Beck Anxiety Inventory: comparative study of patient and non-patient. Korean J Clin Psychol 1997;16:185-197.

34. Vexler A, Tao G, Chen X. A toolkit for clinical statisticians to fix problems based on biomarker measurements subject to instrumental limitations: from repeated measurement techniques to a hybrid pooled-unpooled design. Methods Mol Biol 2015; 1208:439-460.

35. Smith $\mathrm{SM}$, Johansen-Berg $\mathrm{H}$, Jenkinson $M$, Rueckert $\mathrm{D}$, Nichols TE, Miller KL, et al. Acquisition and voxe/wise analy- sis of multi-subject diffusion data with tract-based spatial statistics. Nat Protoc 2007;2:499-503.

36. Buckner RL, Head D, Parker J, Fotenos AF, Marcus D, Morris JC, et al. A unified approach for morphometric and functional data analysis in young, old, and demented adults using automated atlas-based head size normalization: reliability and validation against manual measurement of total intracranial volume. Neuroimage 2004;23:724-738.

37. Jovicich J, Czanner S, Han X, Salat D, van der Kouwe A, Quinn $\mathrm{B}$, et al. MRI-derived measurements of human subcortical, ventricular and intracranial brain volumes: Reliability effects of scan sessions, acquisition sequences, data analyses, scanner upgrade, scanner vendors and field strengths. Neuroimage 2009;46:177-192.

38. Nichols TE, Holmes AP. Nonparametric permutation tests for functional neuroimaging: a primer with examples. Hum Brain Mapp 2002;15:1-25.

39. Smith SM, Nichols TE. Threshold-free cluster enhancement: addressing problems of smoothing, threshold dependence and localisation in cluster inference. Neuroimage 2009;44: 83-98.

40. Hua K, Zhang J, Wakana S, Jiang H, Li X, Reich DS, et al. Tract probability maps in stereotaxic spaces: analyses of white matter anatomy and tract-specific quantification. Neuroimage 2008;39:336-347.

41. Mori SS, Wakana S, van Zijl PCM, Nagae-Poetscher LM. MRI atlas of human white matter. Amsterdam:Elsevier Science; 2005. p. 239.

42. Lancaster JL, Tordesillas-Gutiérrez D, Martinez M, Salinas F, Evans A, Zilles K, et al. Bias between MNI and Talairach coordinates analyzed using the ICBM-152 brain template. Hum Brain Mapp 2007;28:1194-1205.

43. Talairach JJ, Tournoux P. Co-planar stereotaxic atlas of the human brain : 3-dimensional proportional system: an approach to cerebral imaging. Stuttgart:Thieme Verlag;1988. p. 122.

44. Xiao J, He Y, McWhinnie CM, Yao S. Altered white matter integrity in individuals with cognitive vulnerability to depression: a tract-based spatial statistics study. Sci Rep 2015;5: 9738.

45. Choi S, Han KM, Won E, Yoon BJ, Lee MS, Ham BJ. Association of brain-derived neurotrophic factor DNA methylation and reduced white matter integrity in the anterior corona radiata in major depression. J Affect Disord 2015;172: 74-80.

46. Alexander AL, Lee JE, Lazar M, Field AS. Diffusion tensor imaging of the brain. Neurotherapeutics 2007;4:316-329.

47. Henry RG, Oh J, Nelson SJ, Pelletier D. Directional diffusion in relapsing-remitting multiple sclerosis: a possible in vivo signature of Wallerian degeneration. J Magn Reson Imaging 2003; 18:420-426.

48. Müller N. Immunology of major depression. Neuroimmunomodulation 2014;21:123-130.

49. Kim YK, Na KS, Shin KH, Jung HY, Choi SH, Kim JB. Cytokine 
imbalance in the pathophysiology of major depressive disorder. Prog Neuropsychopharmacol Biol Psychiatry 2007;31:10441053.

50. Tarkowski E, Andreasen N, Tarkowski A, Blennow K. Intrathecal inflammation precedes development of Alzheimer's disease. J Neurol Neurosurg Psychiatry 2003;74:1200-1205.

51. Sharief MK, Hentges R. Association between tumor necrosis factor-alpha and disease progression in patients with multiple sclerosis. N Eng/ J Med 1991;325:467-472.

52. Buck D, Hemmer B. Treatment of multiple sclerosis: current concepts and future perspectives. J Neurol 2011;258:17471762.

53. Trapp BD, Nave KA. Multiple sclerosis: an immune or neurodegenerative disorder? Annu Rev Neurosci 2008;31:247-269.

54. McKim DB, Weber MD, Niraula A, Sawicki CM, Liu X, Jarrett $\mathrm{BL}$, et al. Microglial recruitment of IL-1 $\beta$-producing monocytes to brain endothelium causes stress-induced anxiety. Mol Psychiatry 2018;23:1421-1431.

55. Alvez PBG. Inference of a human brain fiber bundle atlas from high angular resolution diffusion imaging. Paris:University of Paris-Sud 11;2011. [Dissertation].

56. Sanjuan PM, Thoma R, Claus ED, Mays N, Caprihan A. Reduced white matter integrity in the cingulum and anterior corona radiata in posttraumatic stress disorder in male combat veterans: a diffusion tensor imaging study. Psychiatry Res 2013;214:260-268

57. Drevets WC, Price JL, Furey ML. Brain structural and functional abnormalities in mood disorders: implications for neurocircuitry models of depression. Brain Struct Funct 2008; 213:93-118.

58. Compare A, Zarbo C, Shonin E, Van Gordon W, Marconi C. Emotional regulation and depression: a potential mediator between heart and mind. Cardiovasc Psychiatry Neurol 2014; 2014:324374.

59. Banks SJ, Eddy KT, Angstadt M, Nathan PJ, Phan KL. Amygdala-frontal connectivity during emotion regulation. Soc Cogn Affect Neurosci 2007;2:303-312.

60. Niogi SN, Mukherjee P, Ghajar J, Johnson CE, Kolster R, Lee $\mathrm{H}$, et al. Structural dissociation of attentional control and memory in adults with and without mild traumatic brain injury. Brain 2008;131(Pt 12):3209-3221.

61. Benedetti F, Poletti S, Hoogenboezem TA, Mazza E, Ambrée $\mathrm{O}$, de Wit $\mathrm{H}$, et al. Inflammatory cytokines influence measures of white matter integrity in Bipolar Disorder. I Affect Disord 2016;202:1-9.

62. Paus T, Zijdenbos A, Worsley K, Collins DL, Blumenthal J, Giedd JN, et al. Structural maturation of neural pathways in children and adolescents: in vivo study. Science 1999;283: 1908-1911.

63. Kemp A, MacMaster FP, Jaworska N, Yang XR, Pradhan S, Mahnke D, et al. Age of onset and corpus callosal morphology in major depression. J Affect Disord 2013;150:703-706.

64. Macmaster FP, Carrey N, Marie Langevin L. Corpus callosal morphology in early onset adolescent depression. J Affect Disord 2013;145:256-259.

65. Xu K, Jiang W, Ren L, Ouyang X, Jiang Y, Wu F, et al. Impaired interhemispheric connectivity in medication-naive patients with major depressive disorder. J Psychiatry Neurosci 2013; 38:43-48.

66. Yuan Y, Hou Z, Zhang Z, Bai F, Yu H, You J, et al. Abnormal integrity of long association fiber tracts is associated with cognitive deficits in patients with remitted geriatric depression: a cross-sectional, case-control study. J Clin Psychiatry 2010; 71:1386-1390.

67. Li JJ, Lu J, Kaur C, Sivakumar V, Wu CY, Ling EA. Expression of angiotensin // and its receptors in the normal and hypoxic amoeboid microglial cells and murine BV-2 cells. Neuroscience 2009;158:1488-1499.

68. Deng Y, Lu J, Sivakumar V, Ling EA, Kaur C. Amoeboid microglia in the periventricular white matter induce oligodendrocyte damage through expression of proinflammatory cytokines via MAP kinase signaling pathway in hypoxic neonatal rats. Brain Pathol 2008; 18:387-400.

69. Kita T, Tanaka T, Tanaka N, Kinoshita Y. The role of tumor necrosis factor-alpha in diffuse axonal injury following fluid-percussive brain injury in rats. Int I Legal Med 2000;113: 221-228.

70. Memon AA, Sundquist K, Ahmad A, Wang X, Hedelius A, Sundquist J. Role of IL-8, CRP and epidermal growth factor in depression and anxiety patients treated with mindfulnessbased therapy or cognitive behavioral therapy in primary health care. Psychiatry Res 2017;254:311-316.

71. Kronfol Z, Remick DG. Cytokines and the brain: implications for clinical psychiatry. Am J Psychiatry 2000;157:683-694.

72. Cho SJ, Kim MK, Bang SY, Bang M, Lee SH. White matter integrity associated with severity reductions in positive symptoms after amisulpride treatment in drug-free patients with schizophrenia. Neurosci Lett 2018;685:131-136.

73. Saito Y, Kubicki M, Koerte I, Otsuka T, Rathi Y, Pasternak O, et al. Impaired white matter connectivity between regions containing mirror neurons, and relationship to negative symptoms and social cognition, in patients with first-episode schizophrenia. Brain Imaging Behav 2018;12:229-237. 